\title{
THE THERMOSPHERE AS A SINK OF MAGNETOSPHERIC ENERGY: A REVIEW OF RECENT OBSERVATIONS OF DYNAMICS
}

\author{
T. L. Killeen \\ Space Physics Research Laboratory, Department of Atmospheric \\ and Oceanic Science, The University of Michigan, Ann Arbor, \\ MI 48109, U.S.A.
}

\section{ABSTRACT}

The study of the dynamics and thermodynamics of the earth's upper atmosphere has made significant progress over the past few years owing to the availability of new global-scale data sets from the Dynamics Explorer satellites. The thermospheric wind and temperature fields at high latitude have been observed to depend strongly on forcing processes of magnetospheric origin. A key momentum source is due to the drag effect of ions convecting in response to electric fields mapped down on the ionosphere from magnetospheric boundary regions. Likewise, an important heat source derives from Joule or frictional dissipation due to ion/neutral difference velocities governed, in turn, by magnetospheric forcing. In this paper we discuss the progress made over the last 2-3 years initiated by the new satellite measurements and we review published data on ion and neutral motions in the context of the energy and momentum coupling between the magnetosphere and the ionosphere/neutral upper atmosphere. The observations indicate the existence of a "flywheel effect" which implies direct feedback from the neutral thermosphere to the magnetosphere vi.a the release of energy and momentum previously "stored" in the neutral thermosphere.

\section{INTRODUCTION}

The past few years have seen an unprecedented influx of new experimental information on the dynamics of the neutral upper atmosphere of the earth. The launch of the Dynamics

Explorer-2 spacecraft/1/ in August of 1981 was a milestone in that satellite instrumentation, for the first time, was capable of directly measuring thermospheric neutral winds on the global-scale. The Fabry-Perot interferometer, FPI, $/ 2 /$ and the wind and Temperature spectrometer, wATS, /3/, /4/ were designed to make independent observations of the meridional and zonal wind components, respectively. These individual component measurements could be combined /5/ to provide a vector representation of the horizontal neutral wind field along the track of the polar orbiting spacecraft. Such vector wind measurements provide key, previously missing, information for studies of the thermospheric response to magnetospheric forcing; a response that occurs through the medium of convecting ionospheric ions set into motion by electric fields of magnetospheric origin.

The main conduits for the flow of mass and energy within the magnetosphere follow, of course, along the geomagnetic field lines. It is clear fxom even a cursory examination of the geometry of the earth's magnetic field that the high latitude region, where field lines converge and pass through the atmosphere and ionosphere, represents an important, fundamental boundary region for the study of magnetospheric processes. The magnetosphere/ionosphere connection has been studied fairly extensively in the past by determining the response of the high-latitude ionosphere to magnetospheric input. For example, the influence of particle precipitation and electric fields on the behavior of the ionosphere has been studied in detail using satellite measurements of ion concentrations and drift velocities as well as ground-based measurements of magnetic field perturbations. Clear relationships have been observed between convecting ions in the high-latitude ionosphere and solar wind parameters indicating the basic magnetospheric control of the ionosphere in this region. Ionospheric signatures with characteristic convection cell geometries have been shown to be dependent on the polarity of the BY and $\mathrm{BZ}$ components of the Interplanetary magnetic field.

This is not the end of the story, however, and it should be remembered that the ionospheric ions are embedded in a relatively sluggish fluid composed of much more numerous (factors of v1000) neutral atoms and molecules. The convecting ions interact collisionally with these neutrals exchanging both momentum and intexnal energy (heat). Similarly, precipitating electrons and ions from the magnetosphere deposit energy into the neutral gas and increase the ionospheric conductivity which, in turn, leads to an enhanced rate of energy interchange between the ions and the neutrals. It is clear that the ultimate sink for much of the 
energy and momentum mapping down from the magnetosphere is in fact the neutral thermosphere whose dynamics have, in the past, received far less attention than their ionospheric counterpart because of basic experimental limitations.

In this contribution, we review the progress made in the last few years using the Dynamics Explorer neutral wind observations (together with other supporting DE-2 data) in elucidating the nature of the coupling between the magnetosphere and the thermosphere via the ionosphere. We refer to recent studies that have shown not only the basic dependency of the high latitude neutral dynamics on magnetospheric forcing but also the complexity of the ionneutral coupling mechanisms which are highly (and non-linearly) dependent on the variable ion-neutral and neutral-ion collision frequencies.

\section{OBSERVATIONS}

The Dynamics Explorer-2 satellite was launched into a polar orbit on $3^{\text {rd }}$ August, 1981, and re-entered the atmosphere on 19th February, 1983. Due to the polar nature of the satellite orbit and to the instrumental requirement for the neutral wind measurements to be made below $\sim 700 \mathrm{~km}$, the high latitude coverage was limited to a set of single local-time-plane "studies", each corresponding to the precession of the perigee location over one or other of the polar regions. Fig. 1 illustrates the geometry of the high latitude observations for a typical South polar study made during October, 1981 with the spacecraft in the 09:00-21:00 hrs 10cal time plane. Because of the separation between the geographic and geomagnetic poles the spacecraft samples different regions of "geomagnetic" space at different Universal Times (UTs) as the geomagnetic pole and its associated ion convection pattern (here depicted by an empirical model due to Heppner, /6/) perform their diurnal revolution about the geographic pole. Thus for the UT depicted in Fig. I, the satellite samples the region associated with sunward ion convection near the dusk auroral zone, whereas at other uTs the satellite samples the magnetic polar cap and the dawn auroral zone as the convection pattern moves with respect to the inertially fixed satellite orbital plane (the diurnal locus of the geomagnetic pole is shown in Fig. 1 by the bold circle). It should also be noted that this diurnal motion of the ionospheric convection region moves the convection pattern with respect to the solar terminator (Fig. 1), a motion which tends to modulate the ion densities within the pattern.

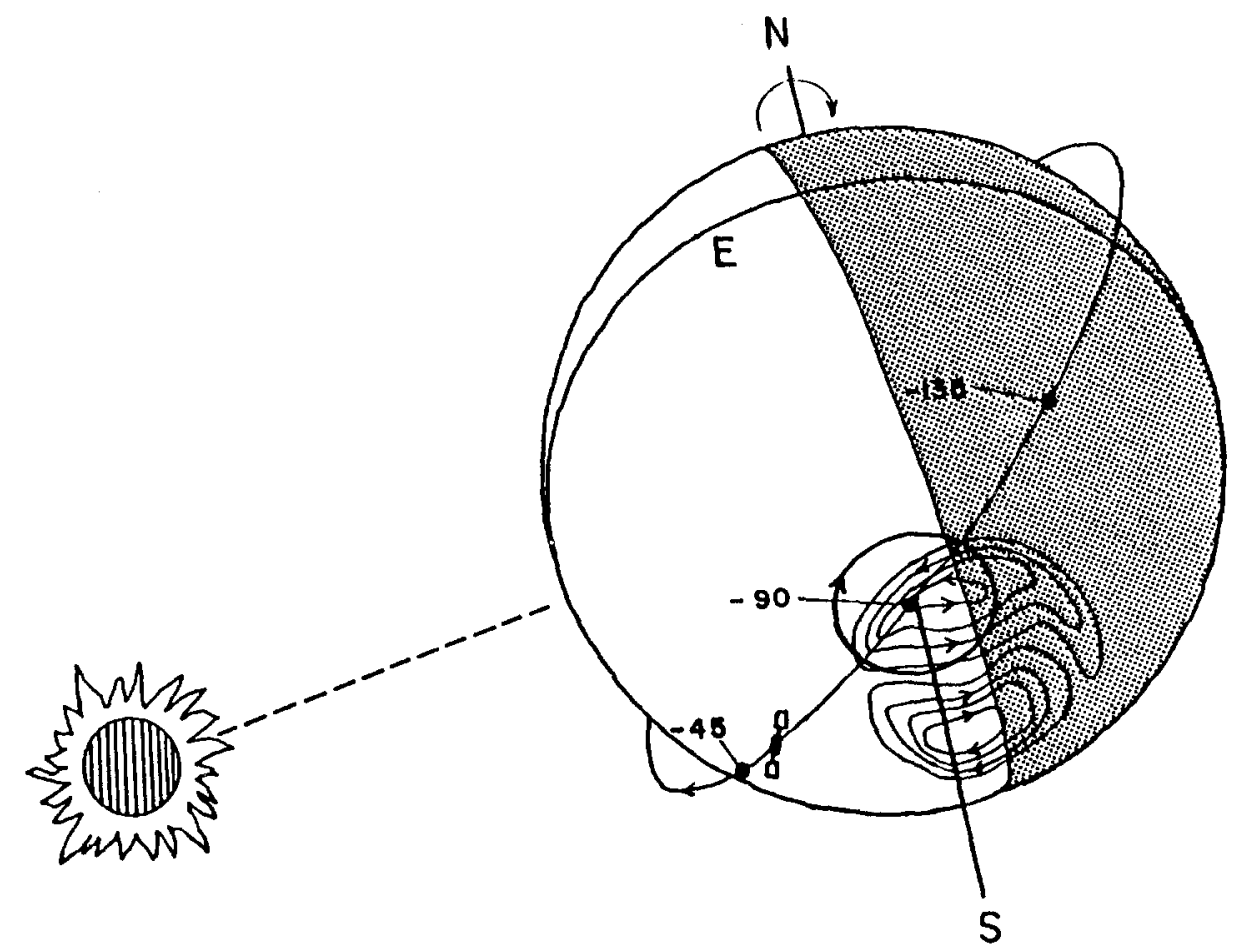

Fig. 1. Schematic showing the geometrical relationship between the DE-2 polar orbit and the high-latitude ion convection pattern for October, 1981 when the spacecraft was in the 09:00/21:00 hrs local time plane with perigee near the south pole. The bold circle represents the diurnal locus of the south geomagnetic pole and the associated convection pattern is represented by a twin-cell circulation similar to that of the Heppner, /6/ empirical model. Note that the diurnal motion of the convection pattern with respect to the fixed orbital plane also moves the region of convection with respect to the solar terminator. 
Since the local time (LT) plane of the orbit precesses slowly ( 4 minutes per day), the data from any given limited time period (days-weeks) are dominated by the "UT effect" due to the orfset poles. This fact can be used to provide an intuitively satisfying picture of the neutral wind circulation at satellite altitude $(\omega 320 \mathrm{~km})$ by replotting data from a set of orbits taken at different UTs (but for the same IT plane, latitude and season) in geomagnetic polar coordinates. Such plots for the southern (summer) hemisphere and the northern (winter) hemisphere are shown in Figs. 2 and 3, respectively (Killeen et al, $15 /$; see also Hays et al, /7/). In both cases, the several orbits, taken together, are suggestive of a twin-cell contra-rotating vortex system in the F-region neutral fluid, analogous to the "classical" ion convection pattern $/ 8 /, 19 /$. This cellular motion in the neutral atmosphere is a response to the ion drag momentum source. It should be cautioned, however, that such pictures are not real "snapshots" of the circulation since the orbits plotted are necessarily separated in time and, as can be inferred from a consideration of Fig. 1, correspond to differing conditions of solar illumination of the convecting ionosphere. To optain a true snapshot of the polar neutral circulation would, of course, require more sampling of the dynamics than possible from a single spacecraft. Limited "snapshots" have been recently compiled from simultaneous $\mathrm{DE}-2$ and Ground-based Fabry-Perot measurements in the northern hemisphere $/ 10 /$ and these confirm the basic pattern shown in Fig. 3 . The consistency between the "synthesized" satellite picture (Fig. 3) and the "snapshots" is evidence, in itself, of a basic, first order, invariance of the neutral circulation pattern in the geomagnetic reference frame.

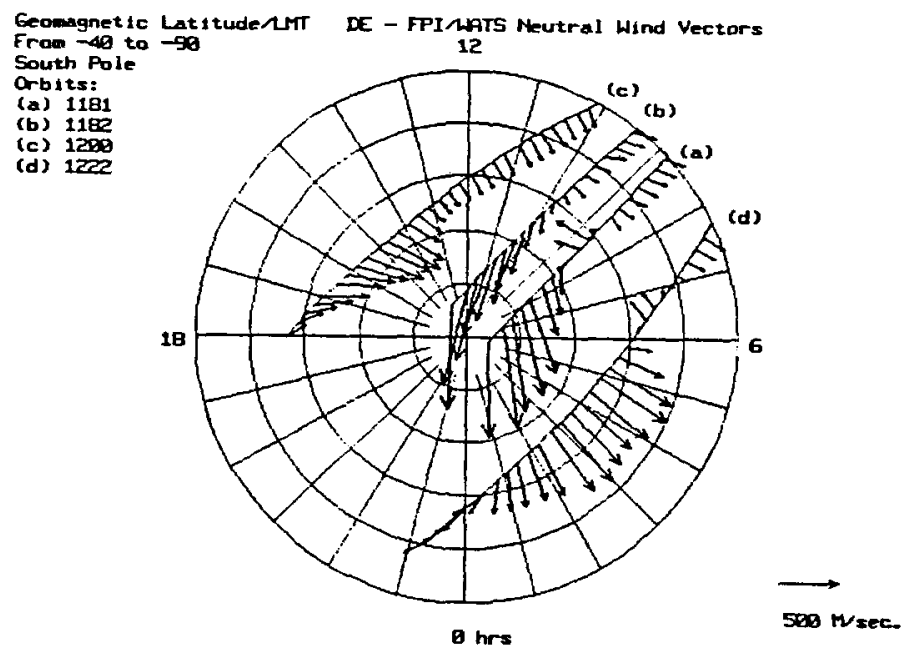

Fig. 2. Geomagnetic polar plot (magnetic latitude and local time) showing four passes of DE-2 neutral wind measurements over the southern hemisphere polar regions. The outer circle is at 40 degree (south) latitude. From Killeen et al, /5/.

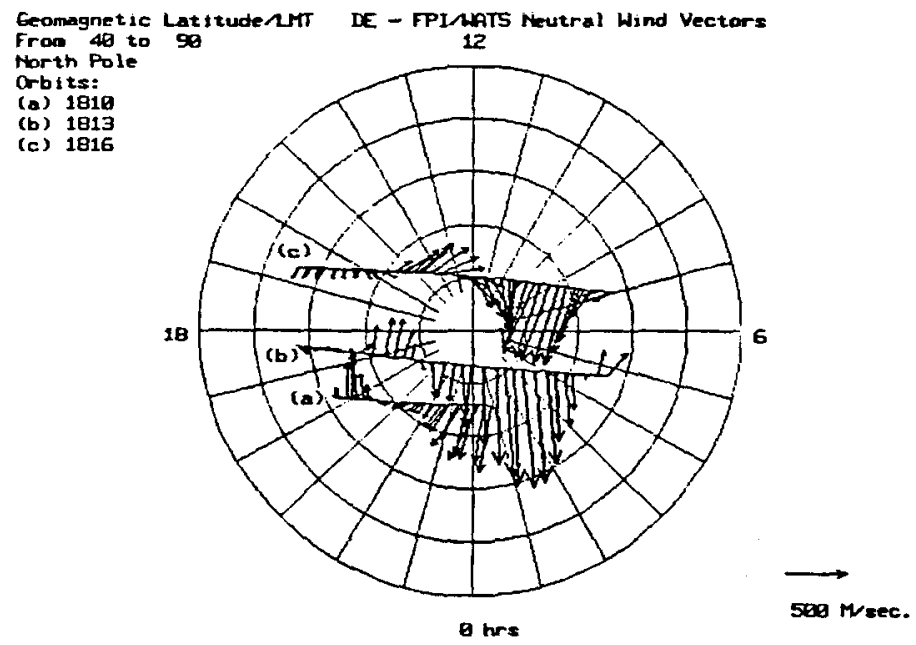

Fig. 3. Same as for figure 2 with the exception that three passes are show for the northern hemisphere polar reqions. 
In addition to the study of individual orbits, a systematic survey of the high latitude thermospheric neutral wind and temperature structure using many tens of orbits from $\mathrm{DE}-2$ has been reported by Hays et al, /7/ (see also, the earlier preliminary study by Killeen et al, /11/). In this work statistical averages of the wind structure for both summer and winter polar regions have been presented and compared with the predictions of the two large TGCMs of Dickinson et al, /12/ and Fuller-Rowell and Rees, /13/. The basic conclusion of this study was that the average polar neutral circulation is best ordered in a geomagnetic reference frame. Fig. 4 from the Hays et al, study/7/ shows four orbits of neutral wind vector information together with a comparison with predictions of the UCL and NCAR TGCMS. As can be seen there is reasonable agreement between the data and theory, at least to first order, in the northern hemisphere data set. The comparisons with theory showed an encouraging level of agreement for the wind data indicating that the models contain much if not all of the basic physics while obviously requiring improved prescriptions for the basic magnetospheric driving functions.

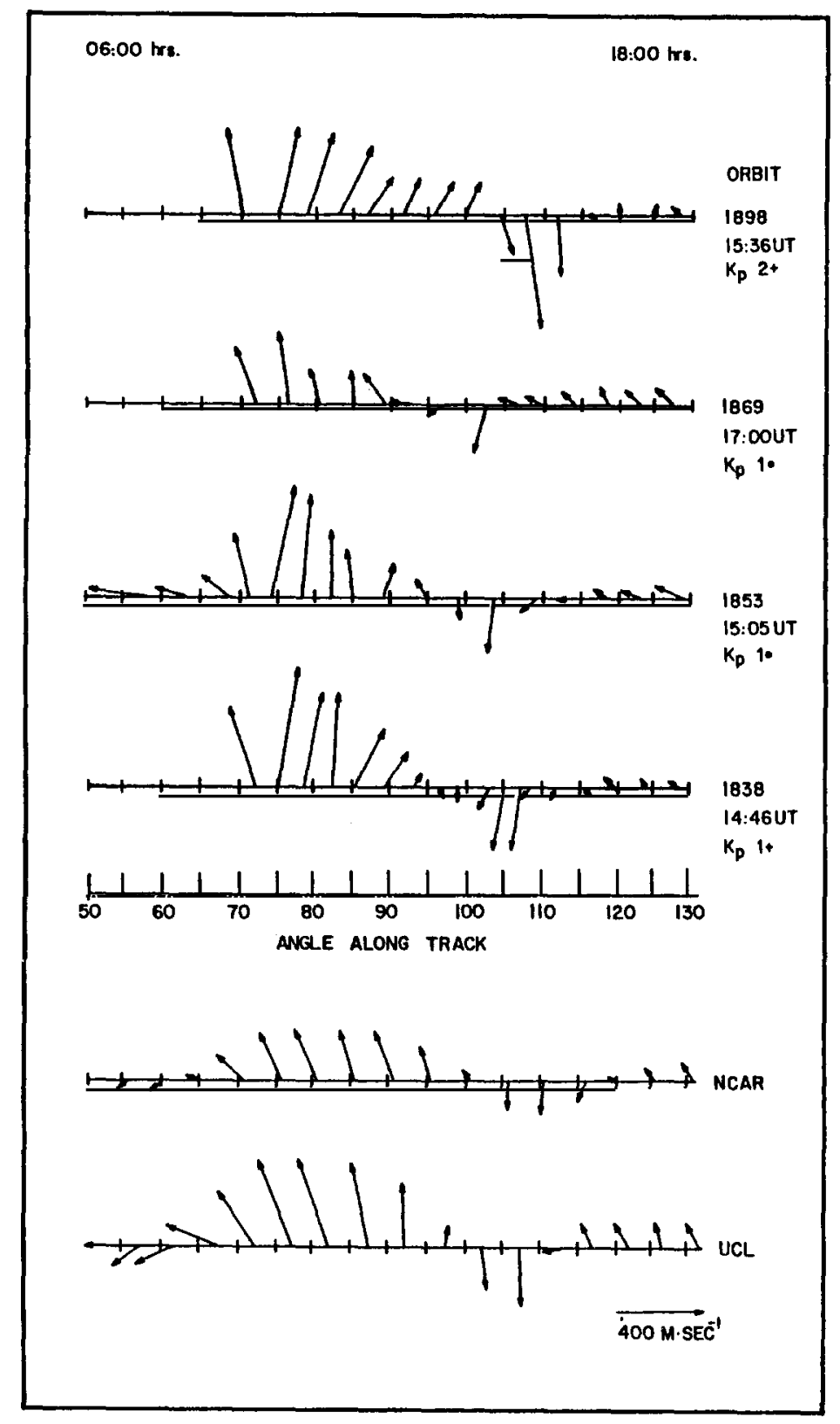

Fig. 4. Neutral wind vectors plotted along the track of DE-2 for four north pole passes from December, 1981 at universal times near 15:00 hrs (top). The satellite crosses the pole from 06:00 LT to 18:00 LT, and the vectors are plotted with respect to this local time plane. Also shown are the model predictions of the NCAR and UCL TGCMS for the same local time plane and universal time conditions (bottom). From Hays et al, $/ 7 /$. 
In Fig. 5 we show a typical output of the TGCM developed at the National Center for Atmospheric Research (NCAR) by R. G. Roble and colleagues as an illustration of the ability of the models to provide global and time-dependent predictions. The figure, taken from a recent paper by Killeen and Roble /14/, illustrates the twin cell vortex in the F-region neutral wind field for the northern hemisphere polar cap using a cross-cap potential of $60 \mathrm{kV}$ and geophysical inputs appropriate to December $12^{\text {th }} 1981$. Four patterns are shown corresponding to $3: 00,9: 00,15: 00$ and $21: 00 \mathrm{hrs}$ UT. The UT effect is evident in the model data as the circulation pattern rotates through a background flow due to solar tidal (day to night) pressure gradients. The satellite/model comparisons have indicated that, on average, the high-latitude thermosphere does behave in a similar fashion to that predicted by theory. The measurements, however, exhibit a much larger degree of variability on an orbit-to-orbit basis (Fig. 4) indicating that the thermosphere has its own "weather" with the variability driven, in large part, by temporal changes in the magnetospheric convection electric fields.

\section{TGCM NEUTRAL WIND PREDICTIONS}
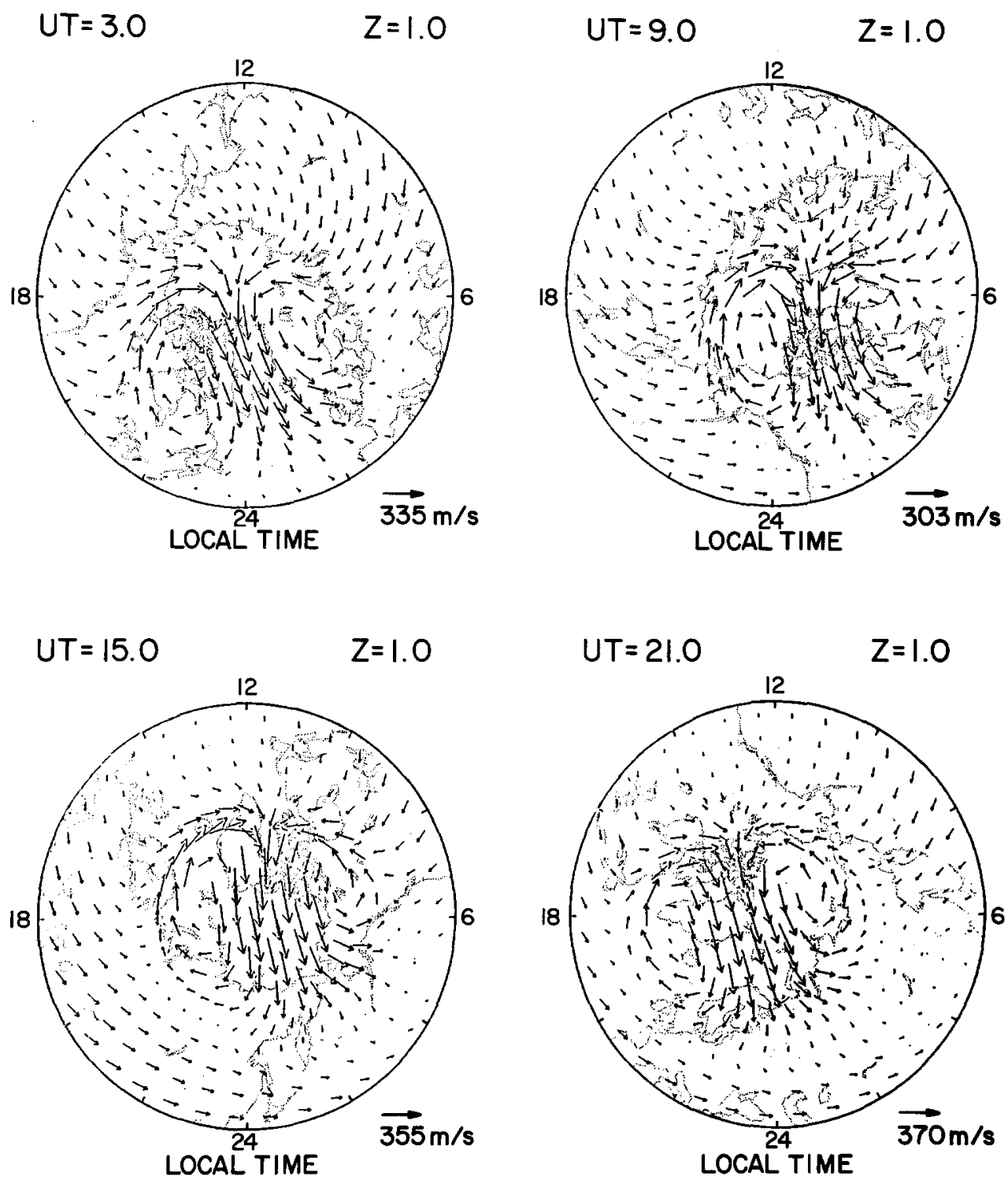

Fig. 5. Global-scale neutral wind predictions of the NCAR TGCM shown for the northern hemisphere and calculated for a diurnally reproducible model run using a cross-polar cap potential of $60 \mathrm{kV}$ and geophysical conditions appropriate to December 12th, 1981. The wind data are calculated for the $z=1$ constant pressure surface equivalent to w320 km. Four universal times are shown. (Figure from Killeen and Roble, /14/). 


\section{ION/NEIJTRAL COUPLING}

The studies referred to above have shown that the neutral thermosphere at high latitudes bears the imprint of ion drag forcing. A close look at the details of the ion/neutral coupling for specific situations using the full range of observables measured by $D E-2$, however, reveals the complexity of the coupling mechanism. In figures 6 and 7 we show data from two passes of DE-2 over the southern hemisphere polar regions separated by a time interval of 3 hours. These data, taken from the study by Killeen et al, /15/, show, from top to bottom, the following measured or calculated parameters: the in-situ horizontal ion drift vector measured using the RPA /16/ and IDM instruments /17/, the in-situ horizontal neutral wind vector measured using the FFI and WAIs instruments, the electron, ion and neutral kinetic temperatures, measured by the Langmuir probe /18/, the RPA and the FPI instruments, respectively, the atomic oxygen and molecular nitrogen concentrations, measured by the NACS instrument /19/ and the electron density, measured by the Langmuir probe. Finally, the bottom panel shows a calculated ion-neutral momentum transfer time constant representing the local e-folding time for the neutral gas to accuire the velocity of the ion gas following a sudden change in the ion drift speed. This time constant, ranging from several tens to hundreds of minutes gives a quantitative measure of the tightness of the ion-neutral momentum coupling. These data have been discussed in detail by Killeen et al, /15/, and here we will only briefly describe the more significant features to illustrate two points, l) the basic complexity of the ion/neutral coupling in the upper atmosohere and 2) possible feedback from the neutral thermosphere to the magnetosphere --- the "flywheel" effect.

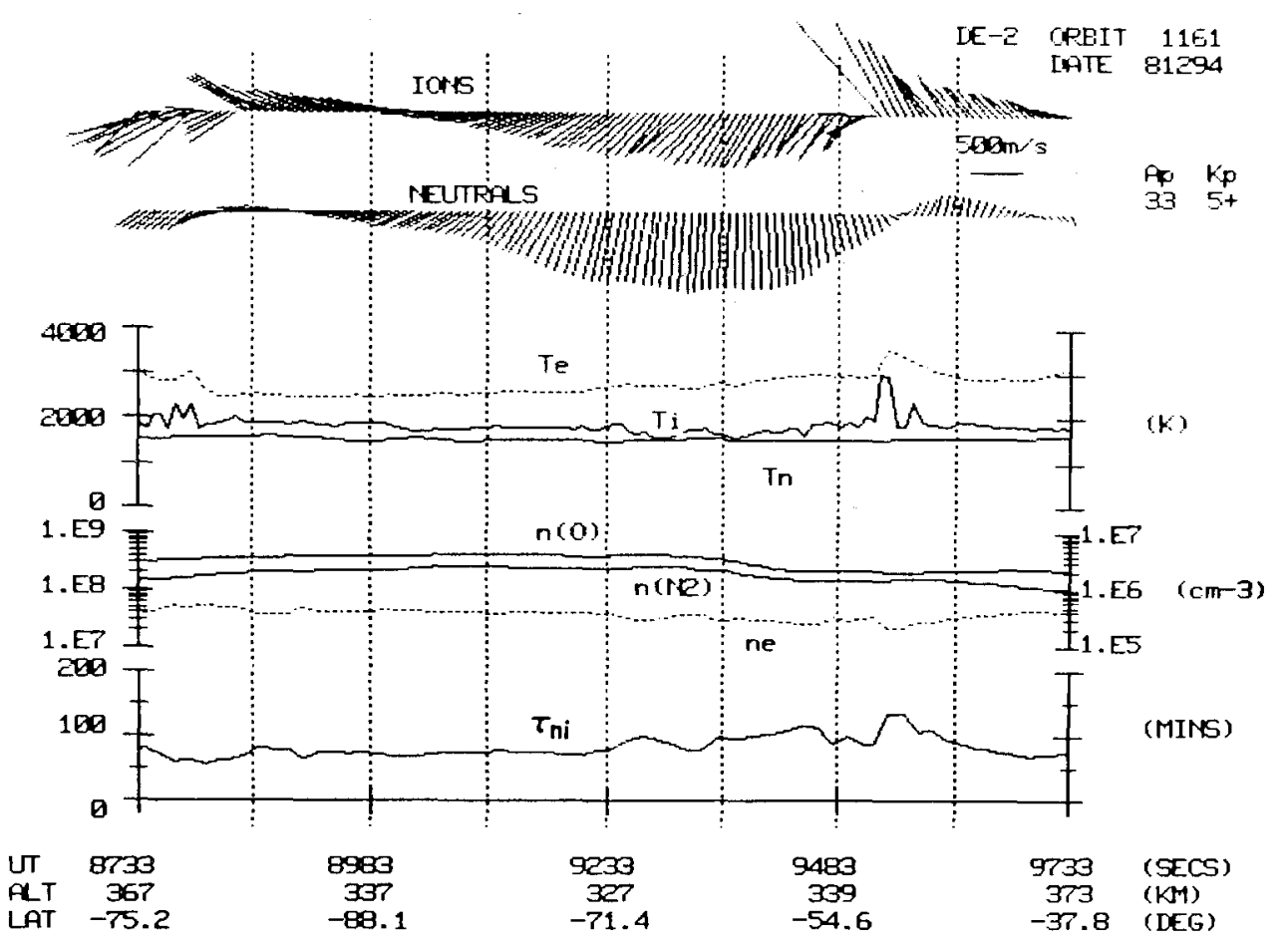

Fig. 6. Geophysical observables measured along the track of DE-2 during orbit 1161 . The ion drifts and the neutral winds are shown in the top two traces plotted against time, altitude and latitude of the spacecraft. The second panel shows the electron, ion and neutral temper atures and the third panel shows the atomic oxygen and molecular nitrogen number densities (left hand scale) and the electron density (right hand scale). The bottom trace shows the calculated ion/neutral coupling time constant. From Killeen et al, /15/.

The first of the two passes, orbit 1161, shown in Fig. 6, depicts a fairly typical situation with both the ions and neutrals exhibiting anti-solar flow over the geomagnetic polar cap bounded by local regions of more sunward flow. There is remarkably good agreement both in magnitude and direction between the independently measured ion and neitral velocities over the dusk-side (left-hand side of figure) and polar cap. The largest ion-neutral difference velocities occur in the dawn-side convection channel, encountered near 9520s UT, where the ions have a high sunward velocity $(\sim 1300 \mathrm{~m} / \mathrm{sec})$ in a region where the neutrals are reversing from anti-sunward to sunward with relatively low velocities. In this convection channel, there is obviously intense frictional (Joule) heating taking place as evidenced most strongly by the enhanced ion temperatures. There is also evidence of a decrease in the ratio of atomic oxygen to molecular nitrogen as the neutral atmosphere responds to the local heat source by upwelling $/ 20 /$ and bringing air rich in molecular nitrogen to satellite altitude. 
Coincident with the convection channel there is also a significant decrease in the electron (ion) density caused, presumably, by an increased rate of recombination due, in part, to the conversion of atomic ions to molecular ions via ion-atom interchange processes that proceed more rapidly at the higher ion temperatures. The molecular ions rapidly dissociatively recombine, leading to a depletion in the ion density. There appears to be a feedback process taking place involving both the heating and the compositional adjustment of the neutral atmosphere to the heating whose net effect is to decouple the ions from the neutrals by effectively lengthening the momentum transfer time constant (bottom panel). As pointed out by Killeen et al, $115 /$, this feedback is not a simple local process but is dependent on the transport of "depleted" ionospheric flux tubes as well as on the height integrated Joule heating. Clearly, to model rigorously such processes will require the self-consistent coupling of a thermospheric dynamical model (TGCM) together with an ionospheric model (e.g. that of Sojka et al, $/ 21 /$. Such comprehensive models are not yet available.

Fig. 7 shows, in an identical format, the measurements made 3 hours later on orbit 1163. It is immediately evident that the ion convection geometry has changed significantly during this interval, although the neutral winds are relatively unchanged. This is taken to be an example of the smoothing or integrating effect of the thermosphere due to the relatively long time constants involved for momentum transfer to the neutrals (10s to 100s rainutes) compared with the much more rapid response (secs) of the ions to changes in the imposed convection electric field. The ion drift velocities indicate relatively turbulent flow with no well-structured anti-solar flow region and with rapid reversals. The frictional heating for this orbit is spread over the entire magnetic polar cap as seen from the enhancements in the ion temperature, the large decrease in the $[0] /\left[\mathrm{N}_{2}\right]$ ratio and the effective ion-neutral momentum decoupling.

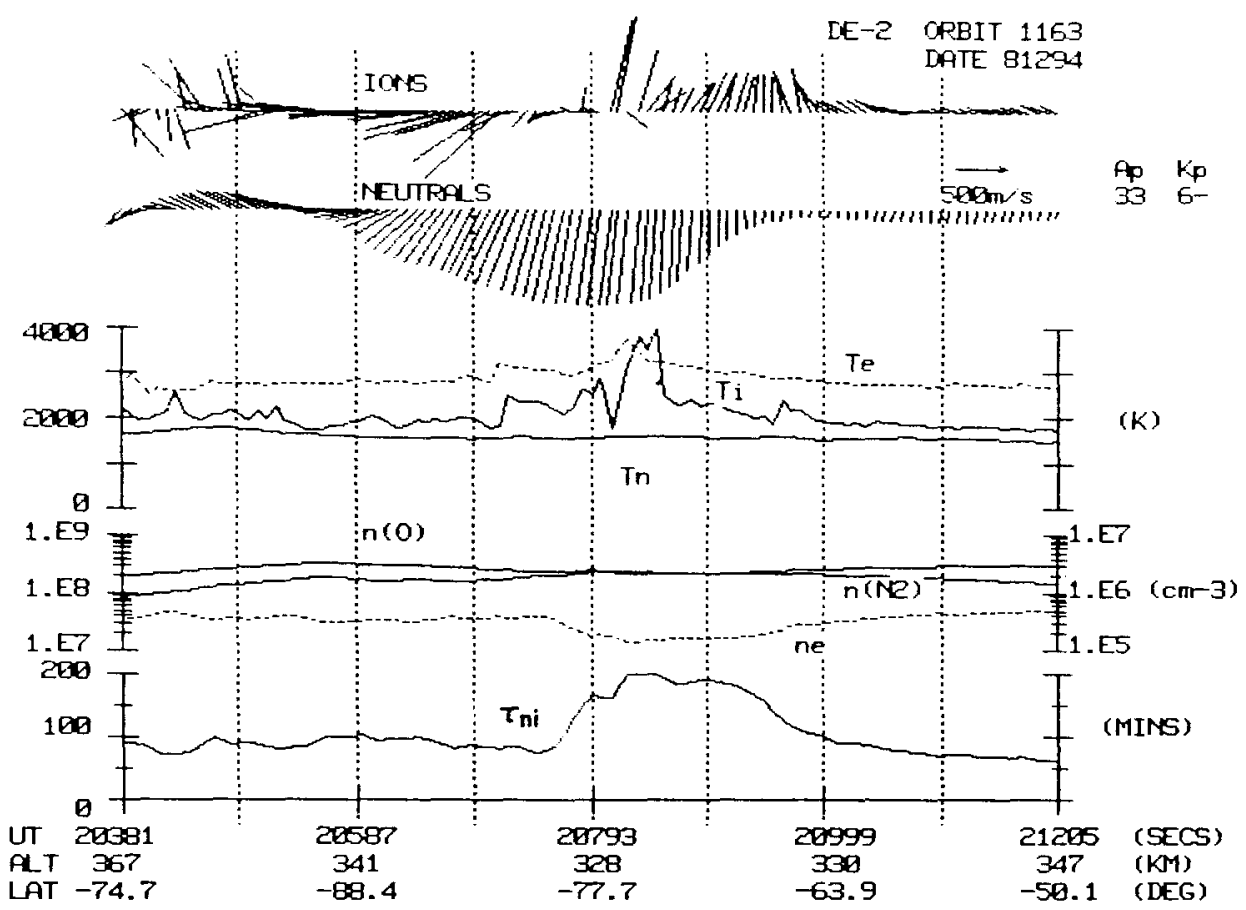

Fig. 7. Same as for Fig. 6 with the exception of orbit 1163.

The two passes, taken together, provide an indication of the existence of a "flywheel" effect in the F-region. It seems clear that for orbit 1161, the ions are driving the neutrals, whereas, for orbit 1163, the neutrals, by reason of their larger inertia, are driving (or, at the very least, impeding) the ions. The simplest interpretation is that the neutral gas, for orbit 1163, has not had sufficient time to adjust to the changed pattern of ion convection and is flowing at a rate and in a direction determined from the previous time history of ion drag forcing. In such a situation the inertia of the neutral gas must have an influence on the whole ionospheric/magnetospheric electrical circuit. It should be pointed out here, of course, that the F-region component of a possible "flywheel" is of much less importance than a postulated E-region component where by far the largest portion of ionospheric conductivity resides. The F-region results are, however, highly suggestive of the existence of such feedback onto the magnetosphere and clearly further experimental and theoretical work is required to put realistic limits on the possible magnetospheric effects of such a process. 
SUMMARY

Satellite measurements over the last few years, such as those illustrated in Figs. 2 and 3 , have confirmed the central importance of ion drag in establishing the high latitude neutral circulation pattern, in agreement with earliex experimental indications from both groundbased (e.g. Hays et al, /22/) and rocket-borne (e.g. Heppner and Miller, /23/) instrumentation. They have also proved to be of use in comparisons with theoretical models $/ 24 /, / 25 /$, $126 /$, enabling a refinement of the parameterizations used for the ion drag momentum source in the large numerical thermospheric general circulation models (TGCMs). Though much work remains to be done to reveal the details of the dependency of the thermospheric dynamics on solar wind parameters, etc, the sateliite measurements have provided useful new constraints for the modelling of thermospheric processes. The data sets have, however, revealed significant complexities that will require the development of coupled ionospheric and thermospheric models to understand fully. Finally, the comprehensive DE-2 measurements have indicated that the neutral thermosphere may indeed, at times, provide some feedback onto the magnetosphere due, basically, to the different time constants for dynamical response of the ionospheric ions and the neutrals to externally imposed momentum sources.

\section{ACKNOWLEDGEMENT}

The work was supported by NASA grant number NAS-5-25691 to the University of Michigan.

\section{REFERENCES}

1. Hoffman, R. A., G. D. Hogan and R. C. Maeh.l, Dynamics Explorey spacecraft and ground operations systems, Space Sci. Instrum., 5, 349-367, 1981.

2. Hays, P. B., T. L. Killeen, and B. C. Kennedy, The Fabry-Perot interferometer on Dynamics Explorer, Space Sci. Instrum., 5, 395-416, 1981.

3. Spencer, N. W., L. E. Wharton, H. B. Niemann, A. E. Hedin, G. R. Carignan, and J. C. Maurer, The Dynamics Explorer wind and temperature spectrometer, Space Sci. Instrum., 5, $417-428,1981$.

4. Spencer, N. W., L. E. Wharton, G. R. Carignan, and J. C. Maurer, Thermosphere zonal winds, vertical motions and temperature as measured from Dynamics Explorer, Geophys. Res. Letts. , 9, 953-956, 1982 .

5. Killeen, T. L., F. B. Hays, N. W. Spencel, and L. E. Wharton, Neutral winds in the polar thermosphere as measured from Dynamics Explorer, Geophys. Res. Iett., 9, 957-960, 1982.

6. Heppner, J. P., Empirical models of high-latitude electric fieläs, J. Geophys. Res., $82,1115-1125,1977$.

7. Hays, P. B., T. L. Killeen, N. W. Spencer, I. E. Wharton, R. G. Roble, B. A. Emery, T. J. Fuller-Rowell, D. Rees, I. A. Frank and J. D. Craven, Observations of the dynamics of the polar thermosphere, J. Geophys. Res., 89, 5597-5612, 1984.

8. Volland, H., A model of magnetospheric electric convection field J. Geophys. Res., Q3, 2695-2699, 1978 .

9. Heelis, R. A., J. K. Lowell, and R. W. Spiro, A model of the high-latitude ionospheric convection pattern, J. Geophys. Res., 87, 6339-6345, 1982.

10. Killeen, T. I., R. W. Smith, P. B. Hays, N. W. Spencer, L. E. Wharton and F. G. McCormac, Neutral winds in the high-latitude winter F-region: Coordinated observations from ground and space, Geophys. Res. Lett., 11, 311-314, 1984a.

11. Killeen, T. I., P. B. Hays, N. W. Spencer, and L. E. Wharton, Neutral winds in the polar thermosphere as measured from Dynamics Explorer, Adv.. Space Res., 2, 10, 133-136, pergamon Press, oxford., 1983.

12. Dickinson, R. E., E. C. Ridley and R. G. Roble, A three-dimensional, general circulation model of the thermosphere, J. Geophys. Res., 86, 1499-1512, 1981.

13. Fuller-Rowell, T. J., and D. Rees, A three-dimensional, time dependent global model of the thermosphere, I. Atmos. SCi., 37, 2545-2567, 1980.

14. Killeen, T. L., and R. G. Roble, Neutral parcel transport in the high-latitude Fregion, Proceedings of the NATO Advanced Workshop on the Morphology and Dynamics of the Polar Cusp, eds A. Egeland and J. A. Holtet, D. Reidel, 1984.

15. Killeen, T. L., P. B. Hays, G. R. Carignan, R. A. Heelis, W. B. Hanson, N. W. Spencer 
and L. H. Brace, Ion-neutral coupling in the high-latitude F-region: Evaluation of ion heating terms from Dynamics Explorer-2, I. Geophys. Res.. 89, in press, 1984b.

16. Hanson, W. B., R. A. Heelis, R. A. Power, C. R. Lippincott, D. R. Zuccaro, B. J. Holt, L. H. Harmon, and S. Sanatani, The retarding potential analyzer for Dynamics Explorer-B, Space Sci. Instrum. , 5, 503-510, 1981.

17. Heelis, R. A., W. B. Hanson, C. R. Lippincott, D. R. Zuccaro, L. H. Harmon, B. J. Holt, J. E. Doherty, and R. A. Power, The Ion Drift Meter for Dynamics Explorer-B, Space Sci. Instrum. , 5, 511-521, 1981

18. Krehbiel, J. P., L. H. Brace, R. F. Theis, W. H. Pinkus, and R. B. Kaplan, The Dynamics Explorer Langmuir Probe Instrument, Space Sci. Instrum., 5, 493-502, 1981.

19. Carignan, G. R., B. P. Block, J. C. Maurer, A. E. Hedin, C. A. Reber, and N. W. Spencer, The neutral mass spectrometer on Dynamics Explorer, Space Sci. Instrum., 5, 429-441, 1981 .

20. Hays, P. B., R. A. Jones, and M. H. Rees, Auroral heating and the composition of the neutral atmosphere, Planet. Space Sci., 21, 559-570, 1973.

21. Sojka, J. J., W. J. Raitt, and R. W. Schunk, A theoretical study of the high-latitude winter F-region at solar minimum for low magnetic activity, J. Geophys. Res., 86 , $609-621,1981$.

22. Hays, P. B., J. W. Meriwether, and R. G. Roble, Nighttime thermospheric winds at high latitudes, J. Geophys. Res., 84, 1905-1913, 1979.

23. Heppner, J. P., and M. L. Miller, Thermospheric winds at high-latitudes from chemical releases, J. Geophys. Res., 87, 1633-1647, 1982.

24. Roble, R. G., R. E. Dickinson, E. C. Ridley, B. A. Emery, P. B. Hays, T. L. Killeen, and N. W. Spencer, The high latitude circulation and temperature structure of the thermosphere near solstice, Planet. Space Sci., 31, 1479-1499, 1983.

25. Roble, R. G., B. A. Emery, R. E. Dickinson, E. C. Ridley, T. I. Killeen, P. B. Hays, G. R. Carignan, and N. W. Spencer, Thermospheric circulation, temperature and compositional structure of the southern hemisphere polar cap during october-November, 1981, J. Geophys. Res., 89, in press, 1984 .

26. Rees, D., T. J. Fuller-Rowell, R. Gordon, T. L. Killeen, P. B. Hays, L. Wharton, and N. W. Spencer, A comparison of wind observations of the upper thermosphere from the Dynamics Explorer satellite with the predictions of a global time-dependent model, Planet. Space Sci., 31, 1299-1314, 1983. 